\title{
Síndrome da fragilidade, um olhar diferenciado para o idoso: relato de experiência
}

\author{
Frailty syndrome, a different look for the elderly: experience report
}

Síndrome de fragilidad, una mirada diferente para los ancianos: relato de experiência

Amanda Cagnani Bonora ${ }^{1 *}$, Maria Laura Verissimo Teixeira ${ }^{1}$, Renata Licursi Nogueira ${ }^{1}$

\section{RESUMO}

Objetivo: Contribuir para área da saúde do idoso, através de um relato de experiência prática, que permitiu o retrato da Síndrome da Fragilidade (SF) e sua correlação com o envelhecimento. Relato de Experiência: O estudo é do tipo exploratório, descritivo, derivado da experiência vivenciada por estudantes, que aplicaram a avaliação global do idoso em uma paciente com 68 anos de idade, coletando dados que foram relacionados $\mathrm{SF}$, permitindo a discussão das características da mesma, e de seus potenciais de riscos. Focando na necessidade de prevenção e orientação da paciente e daqueles não diagnosticados com SF, podendo alterar a morbimortalidade da doença e gerar uma maior qualidade de vida para população idosa. Considerações finais: A experiência ressaltou a importância do olhar integral ao paciente, visto não somente através de dados e exames, podendo, assim, atuar de forma ativa na promoção, prevenção da saúde na comunidade e por fim contribuir para o envelhecimento saudável da população idosa.

Palavras-chave: Assistência integral à saúde, Envelhecimento, Fragilidade.

\begin{abstract}
Objective: Contribute to the area of elderly health, through a report of practical experience, which allowed the portrait of the Frailty Syndrome (SF) and its correlation with aging. Experience Report: The study is exploratory, descriptive, derived from the experience of students, who applied the global assessment of the elderly to a 68-year-old patient, collecting data that were related to SF, allowing the discussion of its characteristics, and its potential risks. Focusing on the need for prevention and guidance for patients and those not diagnosed with FS, which can change the morbidity and mortality of the disease and generate a better quality of life for the elderly population. Final considerations: The experience highlighted the importance of a comprehensive look at the patient, seen not only through data and tests, thus being able to act actively in the promotion, prevention of health in the community and ultimately contribute to the healthy aging of the elderly population.
\end{abstract}

Keywords: Comprehensive health care, Aging, Frailty.

\section{RESUMEN}

Objetivo: Contribuir al área de la salud del anciano, a través de un relato de experiencia práctica, que permitió retratar el Síndrome de Fragilidad (SF) y su correlación con el envejecimiento. Informe de experiencia: El estudio es exploratorio, descriptivo, derivado de la experiencia de estudiantes, quienes aplicaron la evaluación global del anciano a un paciente de 68 años, recolectando datos que se relacionaron con la FS, permitiendo la discusión de sus características, y sus riesgos potenciales. Centrándose en la necesidad de prevención y orientación a los pacientes y no diagnosticados de SF, que pueden cambiar la morbimortalidad de la enfermedad y generar una mejor calidad de vida para la población anciana. Consideraciones finales: La experiencia resaltó la importancia de una mirada integral al paciente, vista no solo a través de datos y pruebas, pudiendo así actuar activamente en la promoción, prevención de la salud en la comunidad y en definitiva contribuir al envejecimiento saludable de las personas mayores. población.

Palabras clave: Atención integral de salud, Envejecimiento, Fragilidad.

${ }^{1}$ Universidade de Franca (UNIFRAN), Franca - SP. *E-mail: amandacbonora@outlook.com 


\section{INTRODUÇÃO}

Nos dias atuais muito tem se estudado sobre o processo do envelhecimento tanto senescente quanto senil, em especial pelo aumento da expectativa de vida que vem modificando a estrutura da pirâmide etária da população mundial e principalmente a brasileira, com aumento crescente de idosos, apontando para um novo perfil demográfico. Pessoas do mundo inteiro estão vivendo mais, e está previsto que em 2050 a população idosa mundial será de aproximadamente 2,1 bilhões (UNITED NATIONS, 2017, SOUSA NFS, et al., 2019).

Esse aumento exponencial da estimativa de vida, tem reformulado em vários aspectos a sociedade, incluindo a área da saúde que até mais da metade do século passado tinha as doenças infecciosas como centro de sua atenção, posto que hoje é ocupado pelas doenças crônicas, mais prevalentes entre os idosos e que, em sua maioria, afetam significativamente a qualidade de vida destes (ORGANIZAÇÃO PANAMERICANA DA SAÚDE, 2012).

Pesquisas europeias e americanas indicam que aproximadamente $20 \%$ dos indivíduos com 70 anos ou mais sofrem perda da autonomia e da capacidade funcional, ficando dependentes para realizar no mínimo uma Atividade de Vida Diária (AVD), como banhar-se, vestir-se, ir ao banheiro e transferir-se, ocasinando a vulnerabilidade e fragilidade do idoso. Condição consequente a diminuição de resistência aos agentes estressores do organismo e da capacidade de retorno à homeostase, gerando, assim, a fragilidade, com seus componentes físicos apoiados no tripé da sarcopenia, desregulação neuroendócrina e alterações imunológicas, provocando, dessa maneira, deficiências em diversos sistemas fisiológicos resultando na Síndrome da Fragilidade (LLANO PMP, et al., 2019; SANTOS PHS, et al., 2015).

No Brasil, apesar de pouco abordada pela literatura brasileira, dados apontam que o número de idosos com dependência funcional esteja entre 19 e $23 \%$ e ao se tratar de idosos restritos ao lar a literatura é significativamente escassa (PAMPOLIM G, et al., 2017). Com isso, a moderna literatura geriátrica classifica esses indivíduos como portadores de Síndrome da Fragilidade (SF), cuja fisiopatologia passou a ser objeto de inúmeros estudos nos últimos anos (PALLIYAGURU DL, et al., 2019).

Estes estudos têm mostrado que a SF é mais prevalente em mulheres, afetadas por doenças crônicas, entre elas diabetes mellitus e hipertensão, depressão, delirium, mal estado nutricional, incontinência urinária, osteoporose, instabilidade postural, além de outros fatores que levam ao aumento da fragilidade como doenças osteomusculares e o avanço da idade (RIBEIRO IA, et al., 2019).

Nesse contexto faz necessário conceituar vulnerabilidade, que muitas vezes é definida como sinônimo de fragilidade. Apesar da mesma estar contida na síndrome não é de sua exclusividade, pois esta é compreendida como um conjunto de fatores coletivos e individuais que resultam em uma grande chance de adoecimento e morte, assim como uma menor possibilidade de enfrentamento diante à vida, é multifatorial, podendo ocorrer pelas mais diversas situações, como moradia, renda, escolaridade, e entre outros, não somente as condições ligadas à saúde, podendo a pessoa vulnerável ser ou não portador de comorbidades independente de sua idade (CARMO ME e GUIZARDI FL, 2018). Ao contrário dos pacientes da SF, que tem seu acometimento e frequência diretamente ligados ao aumento da idade de seus portadores, assim como o número de doenças crônicas associadas (SOUSA NFS, et al., 2019; FERREIRA OGL, et al., 2012; LIBERALESSO TEM, et al., 2017).

Ademais é de igual importância a diferenciação dos conceitos de senescência e senilidade. Assim, o termo senescência, é sinônimo do processo fisiológico de envelhecimento, definido pela organização PanAmericana da Saúde (OPAS) como um processo pertencente a todo ser de uma espécie, que ocorre de modo singular, não reversível, não patológico, de decomposição de um organismo o deixando incapaz de enfrentar o estresse do meio ambiente e assim aumentando suas chances de morte. Em contrapartida, a senilidade traz consigo o aspecto patológico do envelhecimento, onde 0 idoso pode ser afetado por diversas enfermidades, entre elas a Síndrome da Fragilidade (SECRETARIA DE ESTADO DE SAÚDE, 2018).

Assim, o presente trabalho se justifica pelo aumento crescente da população idosa que necessita cada vez mais atenção, além de favorecer informações científicas nas pesquisas brasileiras, corroborando com o 
conhecimento já estabelecido na área. Tendo como objetivo relatar uma experiência prática vivida por acadêmicos, que permitiu correlacionar o envelhecimento com a SF, contribuir para a área da saúde do idoso no quesito do planejamento de medidas profiláticas, desenvolvimento de ações coletivas /individuais, que possibilitem por fim um envelhecimento mais saudável e com qualidade de vida para a população senil.

\section{RELATO DE EXPERIÊNCIA}

O relato exposto é do tipo exploratório, descritivo, derivado da experiência vivenciada pelos alunos do $3^{\circ}$ ano (VI período), durante aulas práticas do Programa de Integração Ensino Saúde da Família (PIESF), módulo componente da grade curricular, presente do primeiro ao oitavo período do curso de medicina. O PIESF VI foi ofertado durante o segundo semestre de 2019, sendo composto pelo eixo temático saúde do idoso, totalizando 24 aulas, divididas entre práticas e teóricas.

A atividade ocorreu durante as visitas dos alunos a um Centro de Integração de Idosos, uma organização da sociedade civil, que presta serviço de assistência social à pessoas idosas, propiciando atividades diversas, como alfabetização, recreação, práticas fisioterapêuticas, esportivas e musicoterapia, através de uma equipe multidisciplinar que busca estimular a independência, autonomia e protagonismo desses idosos nas atividades diárias de vida, sendo este um espaço de convivência e socialização, com atividades programadas e direcionadas de acordo com as condições e necessidades de cada idoso. O grupo de alunos foi orientado a realizar a avaliação global dos indivíduos que lá frequentavam e para tal se organizaram em duplas, sendo cada dupla responsável por uma avaliação.

O indivíduo avaliado, era do sexo feminino, com 68 anos de idade, nível de escolaridade baixo, cursou somente o nível fundamental, tabagista, referiu fazer caminhadas diárias por cerca de 20 minutos, com histórico de depressão e há dois anos uma queda da própria altura, enquanto se levantava para ir ao banheiro de sua casa, onde reside sozinha. Ao exame físico apresentou-se em bom estado geral, anictérica, acianótica, corada, hidratada, com sobrepeso (Índice de Massa Corpórea:30g/m), Pressão Arterial (PA):160sx80 mm/Hg, força nos membros preservadas e sem demais alterações.

Para esta avaliação global, foi usado o protocolo de identificação do idoso vulnerável a partir do Rastreio do Risco de Fragilidade de Idosos (VES-13), um instrumento simples que permite a identificação do idoso vulnerável residente na comunidade, através de sua idade, auto percepção de saúde e da presença de limitações físicas e incapacidades, que toma por base o registro das habilidades necessárias para a realização das tarefas do cotidiano. A paciente avaliada pontuou um total de 1 ponto, classificada como baixo risco para quedas, uma vez que relatou perceber sua saúde como regular.

Além disso quando questionada a respeito de suas atividades diárias, a idosa contou que mesmo após a queda não sofreu limitação em seu cotidiano para atividades básicas de vida diária (Escala de Katz), assim também nas atividades instrumentais como fazer compras ou realizar tarefas domésticas por conta própria (Escala de Lawton Brody), uma vez que não teve nenhuma fratura ou sequela, nem medo de que pudesse cair novamente, contrariando o que ocorre com a maioria dos idosos acidentados. Em acréscimo, o teste de força de preensão manual foi adequado para idade e sexo. Quanto à velocidade de marcha foi possível constatar através de análise e do uso da ferramenta Get up and go que não houve alteração da marcha nem do equilíbrio uma vez que a avaliada se mantinha dentro do escore de normalidade.

Em acréscimo, a mesma não referia perda de peso, apresentando-se com sobrepeso quando avaliada, relatando que havia ganhado alguns quilos nos últimos anos e ao final quando foi questionada a respeito de uma possível sensação de exaustão ou sintoma parecido, a idosa referiu sentir fadiga. Deste modo, obtendo um dos critérios característicos da síndrome. Ademais, foi realizado o Mini exame do estado mental para avaliar sua função cognitiva, através de vários domínios como o de linguagem, orientação espacial e temporal, constatando que a mesma não apresentava declínios cognitivos, obtendo resultado normal, conforme seu nível de escolaridade e idade. O bom estado mental, com a atenção preservada, estado vígil normal e assim como sua tenacidade e concentração foram essenciais para a colaboração e facilidade na aplicação dos testes, com bom entendimento dos resultados e uma boa comunicação entre os alunos e a idosa. 


\section{DISCUSSÂO}

O envelhecimento humano é um processo individual com diferenças e singularidades, sendo difícil delimitar com objetividade e de forma consensual, até mesmo os parâmetros para que um indivíduo seja classificado como idoso. Marcos cronológicos e de expectativa de vida definem que em países pobres e em desenvolvimento são considerados idosos aqueles que têm a partir de 60 anos, já em países desenvolvidos a idade seria 65 anos ou mais. Dessa forma, observou-se que $80 \%$ desta população de indivíduos com 60 anos ou mais é saudável do ponto de vista funcional e cognitivo, sendo considerado o processo de envelhecimento ativo, como a melhoria das oportunidades de saúde e segurança objetivando o aumento na qualidade de vida ao processo que os indivíduos ficam mais velhos (MORAES SA, et al., 2017; MIYAMURA K, et al., 2019).

Considerando assim o envelhecimento saudável presente nos indivíduos que têm preservados sua autonomia, capacidade de tomar decisões, independência para executar as tarefas rotineiras, autocuidado, aceitando as mudanças fisiológicas que ocorrem naturalmente com a idade, mantendo dessa forma uma vida com qualidade. Em contrapartida, uma parcela significativa da população idosa tem condições de saúde precárias as tornando vulneráveis a inúmeros eventos com desfechos adversos (SOUSA NFS, et al., 2018).

Portanto, faz-se necessário o uso da avaliação global do idoso que demanda o uso não só de diversas ferramentas, mas também de uma anamnese e exame físico completo, além do exercício da escuta e compreensão das capacidades e limitações próprias do processo de envelhecimento, que muitas vezes podem ser acentuados pela senilidade para que seja possível avaliá-lo em sua totalidade através dos seus aspectos biopsicossociais (CAMPOS GL, et al., 2020; MINISTÉRIO DA SAÚDE, 2018).

A síndrome ainda não tem marcadores validados, sendo diagnosticada clinicamente, caracterizada por exaustão, fraqueza, perda de peso involuntária, diminuição da velocidade da marcha e do equilíbrio e respectivamente a diminuição da atividade física, podendo gerar diversas consequências como quedas, hospitalização, institucionalização, declínio funcional e até mesmo a morte (BARBOSA KTF, et al., 2017).

Mesmo a queda que parece o menor dos prejuízos causados pela SF tem grande repercussão, uma vez que estudos prospectivos mostram que cerca de $30 \%$ a $60 \%$ da população mundial com idade superior a 65 anos cai anualmente, sendo estas quedas responsáveis por $70 \%$ das mortes acidentais em pessoas com mais de 75 anos (CARLI FVBO, et al., 2019). Além disso, as fraturas em idosos são um obstáculo à saúde pública, estima-se que, no Brasil, elas sejam responsáveis pelas mortes de 10 mil indivíduos por ano e esse cenário é pior nos grandes centros (SOUSA AAS, et al., 2020).

Dessa maneira, um dos aspectos que mais se destacou durante a avaliação foi que, a idosa em questão tinha Hipertensão Arterial Sistêmica (HAS) diagnosticada há mais de 20 anos, tratada com Propanolol e Lozartana, apresentando apesar disso, no decorrer do exame físico, uma pressão arterial divergente, levantando o questionamento sobre a forma que os medicamentos estavam sendo administrados e quanto a eficácia do tratamento. Ao ser feito um levantamento da parte teórica sobre as características próprias da Síndrome da Fragilidade: como sexo feminino, baixo nível de escolaridade, sobrepeso, histórico depressivo, foi pensado a possibilidade da paciente em questão ser portadora da mesma, sendo a idosa classificada como pré-frágil, devido a autopercepção de exaustão (AUGUSTI ACV, et al., 2017; NUNES DP, et al., 2015; RIBEIRO IA, et al., 2019).

A classificação criada por de Fried LP, et al. (2001), baseada em 5 critérios sendo eles perda de peso não intencional; exaustão avaliada por autorrelato de fadiga; diminuição da força de preensão manual; baixo nível de atividade física; e diminuição da velocidade de caminhada classificando, assim, os idosos em três categorias, sendo os com zero critérios considerados não-frágeis, os com um ou dois critérios como préfrágeis e aqueles com três ou mais de cinco dos critérios citados como frágeis (LIBERALESSO TEM, et al., 2017; MELO EMA, et al., 2018).

Os dados obtidos levaram a reflexão de que apesar da importância dos testes, não se deve orientar somente os casos diagnosticados clinicamente por eles, uma vez que a paciente apresentava muitos fatores de risco para a síndrome e fortes chances de evoluir de pré-frágil para frágil no futuro caso não fosse alertada 
dos riscos e tomada as medidas terapêuticas e profiláticas necessárias. Ações que podem evitar outras patologias e suas possíveis consequências como as quedas que podem afetar todo o aspecto biopsicossocial do paciente e de sua família/ambiente que este está inserido, até mesmo podendo cursar em seu declínio funcional e morte (LIBERALESSO TEM, et al., 2017).

No desenvolvimento dessa atividade, houve uma reflexão tanto para os alunos quanto para os idosos, pois foi possível enxergar além de testes e exames o quão necessário é realizar mudanças gradativas e definitivas para o bem-estar destes. Uma vez que, mesmo doenças que parecem isoladas podem culminar em síndromes como a tratada neste artigo, que acaba por afetar toda a vivência e o processo de envelhecimento saudável do idoso.

Por fim, essa experiência foi essencial para ressaltar a importância do olhar integral ao paciente, como um ser complexo e único, visto em todo o seu contexto social, biológico e psíquico, não somente através de dados e exames, podendo, assim, atuar de forma ativa na promoção e prevenção da saúde na comunidade. Dessa maneira, o raciocínio sobre os processos de envelhecimento permitiu elucidar e desmistificar a ideia, de que toda velhice vem necessariamente acompanhada de doenças e limitações, ou seja, senil, mas que também pode ser senescente e ter a profilaxia como peça-chave para esse processo. Ademais, vale destacar que existiu um ganho educacional baseado nas ações implementadas pelos métodos ativos de ensinoaprendizagem, ocorrendo um estímulo crítico-reflexivo que resultará para uma formação mais completa e inovadora gerando profissionais capazes, mais preparados e humanizados.

\section{REFERÊNCIAS}

1. AUGUSTI ACV, et al. Análise da síndrome da fragilidade em idosos na atenção primária - Estudo transversal. Revista Brasileira de Medicina de Família e Comunidade, 2017; 9: 2-8.

2. BARBOSA KTF, et al. Envelhecimento e vulnerabilidade individual: um panorama dos idosos vinculados à estratégia saúde da família. Texto Contexto Enfermagem, 2017; 10: 3-6.

3. CAMPOS GL, et al. A diferença na qualidade de vida entre idosos da zona urbana e rural: uma revisão integrativa de literatura. Revista Eletrônica Acervo Saúde, 2020; Sup59: e4139.

4. CARMO ME, GUIZARDI FL. O conceito de vulnerabilidade e seus sentidos para as políticas públicas de saúde e assistência social. Caderno Saúde Pública, 2018;14:2-10.

5. CARLI FVBO, et al. Ocorrências de quedas em idosos e a polifarmácia. Revista Eletrônica Acervo Saúde, 2019; Sup37; e1002.

6. LIBERALESSO TEM, et al. Prevalência de fragilidade em uma população de longevos na região Sul do Brasil. Saúde e Debate, 2017;10:4-7.

7. LLANO PMP, et al. Factors associated with frailty syndrome in the rural elderly. Revista Brasileira de Enfermagem, 2019; 8:3-5.

8. MELO EMA, et al. Síndrome da fragilidade e fatores associados em idosos residentes em instituições de longa permanência. Saúde Debate, 2018; 13: 3-11.

9. MINISTÉRIO DA SAÚDE. Manual para utilização da caderneta de saúde da pessoa idosa. Brasília-DF, Brasil, 2018; 98: 40-44. Disponível em: https://antigo.saude.gov.br/images/pdf/2019/abril/05/manual-utilizacao-caderneta-pessoa-idosa--9-.pdf. Acessado em: 10 de junho de 2021

10. MIYAMURA K, et al. Síndrome da fragilidade e comprometimento cognitivo em idosos: revisão sistemática da literatura. Revista Latino-Americana de Enfermagem, 2019; 12:2-10.

11. FERREIRA OGL, et a. Envelhecimento ativo e sua relação com a independência funcional. Texto Contexto Enfermagem, 2012; 6:23

12. FRIED LP, et al. Frailty in older adults: evidence for a phenotype. Journals of Gerontology Series A: Biological Sciences and Medical Sciences, Washington, DC, 2001; 56(3): 146-156.

13. SECRETARIA DE ESTADO DE SAÚDE. Governo do Estado do Paraná. Avaliação multidimensional do idoso. Paraná, 2018. Disponível em: https://www.saude.pr.gov.br/sites/default/arquivos_restritos/files/documento/202004/avaliacaomultiddoidoso 2018 atualiz.pdf. Acessado em: 02 de agosto de 2021.

14. MORAES SA, et al. Características das quedas em idosos que vivem na comunidade: estudo de base populacional. Revista Brasileira de Geriatria e Gerontologia, 2017;12: 4-10.

15. NUNES DP, et al. Rastreamento de fragilidade em idosos por instrumento autorreferido. Revista de Saúde Pública, $2015 ; 49: 2$.

16. ORGANIZAÇÃO PAN-AMERICANA DA SAÚDE (OPAS). Conselho Nacional de Secretários de Saúde. O cuidado das condições crônicas na atenção primária à saúde: o imperativo da consolidação da estratégia da saúde da família, 2012. Disponível em: https://bvsms.saude.gov.br/bvs/publicacoes/cuidado_condicoes_atencao_primaria_saude.pdf. Acessado em: 06 de agosto de 2021

17. PALLIYAGURU DL, et al. Índice de fragilidade como um biomarcador de expectativa de vida e saúde: foco em intervenções farmacológicas. Revista Eletrônica Elsevier, 2019; 17:2-8.

18. PAMPOLIM G, et al. Prevalência e fatores associados à dependência funcional em idosos restritos ao lar. Journal of Human Growth and Development, 2017; 9: 2-9.

19. RIBEIRO IA, et al. Síndrome do Idoso Frágil em Idosos com Doenças crônicas na Atenção Primária. Revista da Escola de Enfermagem da USP, 2018:9:2-5.

20. SANTOS PHS, et al. Perfil de fragilidade e fatores associados em idosos cadastrados em uma Unidade de Saúde da Família, Ciência Saúde Coletiva; 2015; 8:3-6.

21. SOUSA AAS, et al. Comorbidades e risco à mortalidade dos idosos pós-cirurgia de fratura de quadril: uma revisão integrativa de literatura. Revista Eletrônica Acervo Saúde, 2020; Sup31: e2939.

22. SOUSA NFS, et al. Envelhecimento ativo: prevalência e diferenças de gênero e idade em estudo de base populacional. Caderno Saúde Pública, 2018;16: 1-7.

23. UNITED NATIONS. World population prospects: key findings and advance tables. The 2017 re-vision. New York: United Nations, 2017. Disponível em: https://www.un.org/development/desa/publications/world-population-prospects-the-2017-revision.html Acessado em 09 de julho de 2021. 